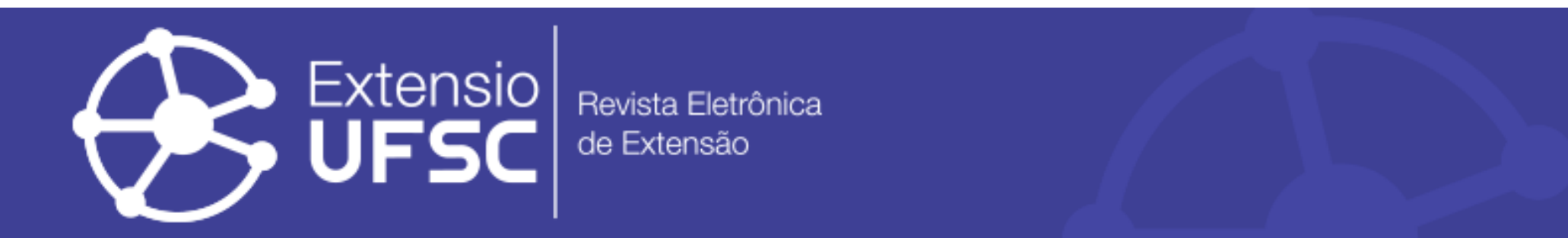

\title{
A LIGA ACADÊMICA DE CLÍNICA E CIRURGIA CARDÍACA: RELATO DE EXPERIÊNCIA
}

\author{
Jefferson Matsuiti Okamoto \\ Universidade Estadual de Ponta Grossa \\ okamotojeff@gmail.com \\ Gabriela Benassi \\ Universidade Estadual de Ponta Grossa \\ gabenassi7@gmail.com \\ Luis Daniel Silva Pilatti \\ Universidade Estadual de Ponta Grossa \\ luisdanielsp@gmail.com \\ Bruna Justus Scheidt \\ Universidade Estadual de Ponta Grossa \\ brunasheidt@gmail.com
}

\author{
Bárbara Zanon da Luz \\ Universidade Estadual de Ponta Grossa \\ barbarazanon@gmail.com \\ Therency Kamila dos Santos \\ Universidade Estadual de Ponta Grossa \\ therencysantos@gmail.com \\ Alessandro Soares Both \\ Universidade Estadual de Ponta Grossa \\ alessandroboth@gmail.com \\ Mário Augusto Cray da Costa
Universidade Estadual de Ponta Grossa
drmarioaugusto@uol.com.br
}

Resumo

A Liga Acadêmica de Cardiologia e Cirurgia Cardíaca (LACCOR) é um projeto extensionista desenvolvido por alunos e professores de Medicina da Universidade Estadual de Ponta Grossa (UEPG) desde 2011. A liga realiza tanto atividades teóricas como práticas na área de cardiologia, proporcionando o contato dos acadêmicos com a iniciação científica, e fornecendo de maneira efetiva ao jovem pesquisador a ampliação dos horizontes. Além disso, possibilita o contato do discente com a comunidade, para que aprenda sobre o território e as pessoas que ali vivem, fortalecendo vínculos e aprendendo desde o início sobre a relação médico-paciente. É crucial que os acadêmicos dos cursos de saúde estejam preparados para enfrentar a nova realidade epidemiológica, por meio de pesquisas e atividades desenvolvidas que habilitarão esses futuros profissionais no cuidado as disfunções cardiovasculares. Até o momento, as atividades demonstraram que o estudante pode ser um agente de transformação social, assimilando os benefícios da iniciativa, do voluntariado e o quanto isso pode fazer a diferença em comunidades a margem da sociedade sem assistência em saúde adequada.

Palavras-chave: Ligas Acadêmicas. Cardiopatias. Extensão Universitária.

\section{AN EXTENSION PROJECT EXPERIENCE: THE ACADEMIC LEAGUE OF CLINIC AND HEART SURGERY}

\begin{abstract}
The Academic League of Cardiology and Heart Surgery (LACCOR) is an extension project developed by students and teachers of Medicine from Ponta Grossa State University (UEPG) since 2011. The league provides the contact of academics with scientific initiation in the area of cardiology, effectively providing the young researcher with broader horizons. In addition, it allows the student's contact with the community, so that he learns about the territory, the people who live there, strengthening ties and learning from the beginning about the doctor-patient relationship. It is crucial that academics in health care courses are prepared to face the new epidemiological reality through research and activities developed since graduation that will enable these future health professionals to care for cardiovascular functions. Until now, the activities demonstrated that the student can be an agent of social transformation, assimilating the benefits of initiative, volunteering and how much this can make a difference in marginalized communities without adequate health care.

Keywords: Academic Leagues. Heart Disease. University Extension.

\section{EXPERIENCIA DE UN PROYECTO DE EXTENSIÓN: LA LIGA ACADÉMICA DE CLÍNICA Y CIRUGÍA CARDÍACA}

Resumen

La Liga Académica de Cardiología e Cirurgia cardiaca (LACCOR) es um projeto extensión ISTA desenvolvido por estudiantes y profesores de Medicina de la Universidad Estatal de Ponta Grossa (UEPG) desde 2011. La liga proporciona o contato do mesmo com iniciación científica en el área de la cardiología, proporcionado de maneira efetiva ao joven 
investigador uma ampliación de los horizontes. Además, posibilita el contacto del alumnado com la comunidad, para que aprenda sobre el territorio y las personas que allí viven, fortaleciendo vínculos y aprendiendo desde el inicio sobre la relación médico-paciente. Es crucial que los académicos de los cursos de salud estén preparados para enfrentar la nueva realidad epidemiológica, a través de investigaciones y actividades desarrolladas que habilitan los futuros profesionales en el cuidado con las disfunciones cardiovasculares. Hasta ahora, las actividades demostraron que lo estudiante puede ser un agente de transformación social, asimilando los beneficios de la iniciativa, del voluntariado y cuánto puede hacer la diferencia en comunidades al margen de la sociedad sin asistencia en salud adecuada. Palavras clave: Liga académica. Cardiopatías. Extensión Universitaria. 
A liga acadêmica de clínica e cirurgia cardíaca: relato de experiência

\section{INTRODUÇÃO}

Ligas acadêmicas relacionadas à saúde podem ser descritas como organizações estudantis que desenvolvem para seus membros oportunidades de atividades que atendam ao tripé ensino, pesquisa e extensão, abrangendo uma determinada área da saúde, visando seu aprendizado e desenvolvimento, sendo gerida pelos próprios estudantes, sob orientação de docentes (PÊGOFERNANDES; MARIANI, 2011; HAMAMOTO FILHO et al., 2011; COSTA et al., 2009; MONTEIRO et al., 2008; NEVES et al., 2008).

Assim um dos principais objetivos de uma liga acadêmica médica é fornecer as ferramentas necessárias para que o aluno seja um agente de transformação social (HAMAMOTO FILHO, 2011). A liga proporciona isso na medida em que estabelece o contato direto entre estudante e comunidade, para que aprenda sobre o território de atuação e as pessoas que ali vivem, fortalecendo vínculos e estimulando desde o início sobre a relação médico-paciente (ABLAM, 2010; GADAMER, 1994). Baseada nisso, a Liga Acadêmica de Cardiologia e Cirurgia Cardíaca é um projeto de extensão desenvolvido por alunos e professores do curso de medicina da Universidade Estadual de Ponta Grossa, com início em 2011 que surge para debater e intervir em doenças cardiovasculares e seus respectivos fatores de risco cada vez mais presentes no cotidiano do brasileiro.

No Brasil, seguindo a tendência mundial, evidencia-se uma situação de transição epidemiológica, observando-se uma alteração da mortalidade decorrente de doenças infectocontagiosas e materno-infantis, pela mortalidade advinda de causas externas e disfunções classificadas como de origem crônico-degenerativas (PEREIRA; ALVES-SOUZA; VALE, 2015).

Contribuindo para essa nova realidade os seguintes fatores: transição demográfica, com queda da taxa de natalidade e aumento do número de idosos (SILVEIRA NETTO, 2000); mudanças no estilo de vida da população, com alterações nos hábitos alimentares, crescente oferta de alimentos industrializados e calóricos; redução generalizada da atividade física levando ao aumento do número de indivíduos com excesso de peso (WHO, 2010). Em 2000, a Organização Mundial da Saúde (OMS) estimou que o número de pessoas com sobrepeso chegará a dois bilhões em 2030. Sendo que a prevalência mundial da obesidade quase dobrou entre 1980 e 2008. Atualmente existem cerca de 250 milhões de pessoas nessa condição, sendo vários os países que sofrem com essa epidemia, inclusive o Brasil (WHO, 2010).

Posta essa realidade, houve um aumento substancial das doenças e agravos não transmissíveis, que constituem um variado número de eventos mórbidos como o diabetes mellitus (SILVEIRA NETTO, 2000). Dados obtidos da Federação Internacional de Diabetes 
A liga acadêmica de clínica e cirurgia cardíaca: relato de experiência

(IDF, 2009) indicam que o diabetes mellitus (DM) afeta pelo menos 285 milhões de pessoas no mundo e espera-se um aumento desse número para 438 milhões em meados de 2030 (GUARIGUATA et al., 2011). Sendo que no Brasil, foi verificada uma prevalência de DM na população adulta de 7,6\% (SBD, 2015). Nessa perspectiva, a inadequação da dieta associada ao sedentarismo são importantes fatores desencadeantes de doenças metabólicas, de tal maneira que a epidemia mundial de obesidade está diretamente relacionada ao número elevado de casos de diabetes mellitus tipo 2 (COELHO; BURINI, 2009; MARIATH et al., 2007; PEREIRA; FRANCISCHI; LANCHA JR, 2003).

Dados da OMS demonstram que os principais fatores de riscos - obesidade abdominal, diabetes mellitus, dislipidemia, estresse, depressão, hipertensão arterial, tabagismo e sedentarismo - estão claramente associados ao desenvolvimento de doenças cardiovasculares, o que consequentemente instiga à maior ocorrência de acidente vascular encefálico e infarto agudo do miocárdio (WHO, 2005).

Assim, ao analisarmos a epidemiologia das doenças cardiovasculares, observamos que têm o mesmo comportamento neste início de século que tinham as grandes endemias dos séculos passados. Isto fica claro observando os dados da OMS nas últimas décadas: das 50 milhões de mortes, as doenças cardiovasculares foram responsáveis por 30\% desta mortalidade, ou seja, 17 milhões de pessoas (BEAGLEHOLE; BONITA, 2008; BUTTLER, 2011).

Nesse entender, visto que o Brasil está incluso nessa projeção, é fundamental o preparo e qualificação da saúde para assistir essa demanda. Sendo necessários projetos, como ligas acadêmicas médicas, que preconizam pela integralidade das ações, unindo pesquisa, ensino e extensão desde a graduação a fim de capacitar o profissional da saúde acerca de disfunções cardiovasculares. Esse artigo visa relatar a experiência da LACCOR, demonstrando as metas e analisar os resultados das atividades realizadas

\section{MATERIAIS E MÉTODOS}

A LACCOR é um projeto extensionista desenvolvido por alunos e professores de Medicina da UEPG. Com início em 2011, conta com 40 integrantes discentes, em sua maioria integrantes dos diversos níveis de graduação do curso de medicina da UEPG, tendo como coordenador um cardiologista, professor na instituição; com a eventual participação de professores de outras especialidades, dependendo da atividade teórico-prática desenvolvida.

Atualmente, para consagrar-se como membro efetivo da LACCOR, basta o aluno estar regularmente matriculado em cursos de medicina do país. Sendo que o acadêmico deve 
A liga acadêmica de clínica e cirurgia cardíaca: relato de experiência

obrigatoriamente comparecer à primeira reunião anual da liga e obter mais de $75 \%$ de presença nas reuniões científicas. Uma vez integrados à LACCOR os alunos têm acesso a todas as atividades da liga que queiram participar. Sendo algumas delas de caráter obrigatório para sua permanência como membros efetivos da LACCOR. Essas atividades têm como base atender ao tripé do ensino-pesquisa-extensão.

Para realização das atividades foram estabelecidos horários extracurriculares para que não afetassem a grade curricular básica. Posto que os alunos devem dedicar ao menos quatro horas quinzenais ao projeto, duas horas em alguma atividade desenvolvida para comunidade como na Vila Social Tio Barros ou na Instituição Educacional Duque de Caxias, e às duas horas restantes em reuniões científicas quinzenais realizadas em uma clínica de cardiologia.

É de caráter obrigatório para os discentes participar de pelo menos uma atividade de extensão. Aqueles que desejaram participar de projetos de pesquisa na área da cardiologia ou cirurgia cardíaca receberam todo respaldo da liga, sendo hoje desenvolvido um número significativo de estudos na área por membros da LACCOR.

\section{RESULTADOS E ANÁLISE}

Apesar de alguns avanços no campo social ainda deparamos em nosso município com uma grande parcela da população vivendo abaixo da linha da pobreza. Esses indivíduos têm sua sobrevivência amparada por instituições não governamentais que buscam com recursos da comunidade promover humana e socialmente essas pessoas carentes em todos os aspectos, necessitadas de amparo na área de alimentação, vestuário, moradia, educação, higiene e saúde.

A OMS define saúde como sendo "estado de completo bem-estar físico, mental e social, e não simplesmente à ausência de doença ou enfermidade - é um direito fundamental, e que a consecução do mais alto nível de saúde é a mais importante meta social mundial, cuja realização requer a ação de muitos outros setores sociais e econômicos, além do setor saúde".

Contudo, partindo da análise do conceito da saúde da OMS (1946), Ferrara (1972) propõe um novo conceito, no qual a saúde é concebida como "o contínuo agir do ser humano ante o universo físico, mental e social em que vive, sem regatear um só esforço para modificar, transformar e recriar aquilo que deve ser mudado". Assim, atribuem ao conceito uma dimensão dinâmica, valorizando o papel dos seres humanos na manutenção e na transformação da saúde (individual e coletiva), colocando-os como atores sociais do processo da própria vida (BRÊTAS; GAMBA, 2006). 
A liga acadêmica de clínica e cirurgia cardíaca: relato de experiência

Destarte, em nossas atividades extensionistas damos a oportunidade ao acadêmico de provar o seu valor e o quanto o mesmo pode ser um agente de transformação social, demonstrando os benefícios da iniciativa, do voluntariado e o quanto isso pode fazer a diferença em comunidades a margem da sociedade sem assistência em saúde adequada.

\subsection{Vila Social Tio Barros}

O foco do projeto é atuar na área de promoção da saúde cuja estratégia é uma das fundamentais do programa, contribuindo assim para a construção de ações que possibilitam assistir as necessidades sociais em saúde e salientar os aspectos que determinam o processo saúde-adoecimento como: violência, desemprego, subemprego, falta de saneamento básico, habitação inadequada e/ou ausente, dificuldade de acesso à educação, fome, urbanização desordenada, qualidade do ar e da água ameaçada e deteriorada.

Atualmente existem seis famílias integrantes, com média de três a quatro pessoas por grupo familiar. Sendo que os discentes prestam atendimento a essas famílias, bem como a outros membros da comunidade vizinha no bairro do Jardim Maracanã, que frequentam as reuniões realizadas pelo projeto aos sábados.

Os alunos foram sempre supervisionados pelos professores envolvidos no projeto, sendo as visitas médicas feitas preferencialmente durante os finais de semana. Assim, na presença de alguma patologia, os médicos envolvidos no projeto instituíram tratamento ou encaminharam os assistidos pelo sistema único de saúde. Além disso, os acadêmicos fizeram acompanhamento da evolução da doença com visitas diárias ou mais espaçadas de acordo com a gravidade da patologia, mantendo os professores informados das evoluções, intervindo de acordo com a necessidade. E no campo da prevenção de doenças e promoção à saúde, foram feitas duas palestras mensais aos sábados voltadas à higiene e prevenção de doenças, as palestras duravam de 30 a 40 minutos, apresentadas de forma simples e didática.

\subsection{Instituição Educacional Duque de Caxias}

A Instituição Educacional Duque de Caxias conta hoje com aproximadamente 350 crianças em sua unidade localizada no Jardim América na cidade de Ponta Grossa. Os discentes da LACCOR prestam assistência em saúde sob supervisão dos docentes. Sendo que, um dos principais projetos desenvolvidos é o de acuidade visual, sob coordenação de um oftalmologista, no qual os acadêmicos realizam a triagem oftalmológica através do teste de Snellen. A tabela de Snellen contém linhas com letras que começam maiores em cima e vão diminuindo de tamanho para baixo, ou símbolos utilizados para pessoas não alfabetizadas ou crianças pequenas, sendo a 
tabela posicionada a uma distância de 6 metros. Quando a criança conseguia distinguir bem até a $8^{a}$ linha, sua visão era considerada dentro dos padrões da normalidade. Porém, caso não fosse além da $4^{a}$ linha, ou obtivesse dois erros em qualquer linha entre a quarta e oitava, a criança era encaminhada aos cuidados de um oftalmologista, pois estava com perda de capacidade visual e precisaria de lentes de correção.

É sabido que a realização do exame de rotina de acuidade proporciona uma maior identificação das crianças que necessitam de atendimento oftalmológico, melhorando a saúde visual, colaborando na atenuação dos elevados índices de evasão escolar ou repetência e prevenindo diversas complicações oculares de maior âmbito. Além disso, familiariza o estudante com o exame visual.

Infelizmente, um dos problemas encontrados na vida da criança em idade escolar é a deficiência visual. Do ponto de vista da saúde pública, é muito dispendiosa e mesmo inexequível a investigação de problemas oculares em crianças, por oftalmologistas, em exame de massa. Dessa maneira, a solução é uma aplicação de triagem oftalmológica por pessoal não médico, treinado e supervisionado.

Prezando pela integralidade da atenção em saúde também é realizado exame físico geral nos alunos do instituto e palestras em saúde, tanto para as crianças e adolescentes, quanto para os pais.

\subsection{Reuniões científicas na clínica de cardiologia}

As reuniões foram realizadas quinzenalmente, com duração de duas a três horas, às segundas-feiras, tendo como palestrantes doutorandos do sexto ano ou pesquisadores convidados. Quando os doutorandos palestravam, os temas abordados eram casos clínicos de pacientes internados, devido a complicações cardíacas, na enfermaria do Hospital Santa Casa de Misericórdia de Ponta Grossa. Na palestra era mandatório que se estabelecesse uma correlação entre a fisiopatologia da doença, o quadro clínico, meios diagnósticos, tratamento, prognóstico e prevenção. Sempre montando um caso que instigasse a curiosidade e o pensar clínico dos ouvintes. Por fim, pode-se observar uma evolução por parte dos acadêmicos, como profissionais da saúde, os alunos tornam-se aptos a diagnosticar, tratar e orientar os pacientes quanto à prevenção de possíveis complicações de doenças cardiovasculares, gerando um melhor atendimento e beneficiando diretamente a população cardiopata, que é melhor assistida. 
A liga acadêmica de clínica e cirurgia cardíaca: relato de experiência

\subsection{Iniciação científica}

$\mathrm{Na}$ área da pesquisa, os acadêmicos realizaram projetos de iniciação científica e trabalhos de conclusão de curso sob coordenação dos professores participantes.

Segue abaixo algumas linhas de pesquisa em desenvolvimento por membros da LICCOR:

\begin{tabular}{|c|}
\hline $\begin{array}{c}\text { Impacto da lesão carotídea na base de cálculos EuroSCORE em pacientes } \\
\text { coronarianos }\end{array}$ \\
\hline Fatores associados com o desenvolvimento da dor crônica pós-esternotomia \\
\hline Avaliar tempo de cirurgia, tempo de radiação e complicações do implante de \\
marcapasso, por meio de uma técnica padronizada. \\
\hline Prevalência de fibrilação atrial em pacientes portadores de marcapasso \\
\hline Taxa de perviedade precoce dos enxertos após endarterectomia coronariana \\
\hline Função ventricular esquerda após cirurgia valvar
\end{tabular}

Tabela 1 - Projetos de pesquisa em desenvolvimento

Fonte: Os Autores

O contato com a iniciação científica proporciona de maneira efetiva ao jovem pesquisador a ampliação dos horizontes, podendo ir além do processo puramente informativo que muitas vezes está limitado na sala de aula. Tendo a oportunidade de exercer o pleno raciocínio pela via do método científico. Bem como de praticar o exercício da criatividade científica, satisfação da curiosidade intelectual, aperfeiçoamento do espírito crítico, aprendizado e consolidação de outros conhecimentos necessários à complementação da sua formação.

\section{CONSIDERAÇÕES FINAIS}

Visto que as doenças cardiovasculares são a maior causa de óbitos no mundo, tem-se a importância de uma liga tanto com fins curativos quanto preventivos para doenças cardiovasculares e suas comorbidades. Portanto é crucial que os acadêmicos dos cursos de saúde, em especial os alunos do curso de medicina, estejam preparados para enfrentar essa nova realidade epidemiológica, através de pesquisas e atividades desenvolvidas desde a graduação, a fim de habilitar esses futuros profissionais de saúde acerca das disfunções cardiovasculares.

Vale ressaltar que o intuito de uma liga acadêmica médica é instruir o aluno a fim de que ele seja um agente de transformação social. Nessa perspectiva por intermédio da Liga de Cardiologia e Cirurgia Cardíaca foi possível proporcionar aos membros efetivos experiências únicas que colocaram em prática a relação médico-paciente, além dos conhecimentos teóricos 
A liga acadêmica de clínica e cirurgia cardíaca: relato de experiência

aprendidos por meio das palestras e o desenvolvimento do raciocínio clínico para diagnóstico de doenças cardiovasculares. Proporcionando uma assistência adequada as famílias beneficiadas pelas instituições já citadas, que tiveram a chance de tirar suas dúvidas e receberem orientações acerca de questões ligadas à saúde.

Desse modo, encerra-se enfatizando que saúde é um conceito dinâmico, essa comunidade amparada deve ser permanentemente atendida, pois carece em muitos aspectos e precisa de apoio continuado. Sendo que os resultados das atividades da LACCOR serão observados a longo prazo com a formação de melhores profissionais de saúde e provável melhora nos indicadores de saúde da região.

\section{REFERÊNCIAS}

ABLAM. Diretrizes Nacionais de Ligas Acadêmicas de Medicina. São Paulo: [s. n.], 2010. Disponível em:< http://ablam.org.br/diretrizes-nacionais/ >. Acesso em: 20/12/2016.

BEAGLEHOLE R.; BONITA R. Global publichealth: a scorecard. Lancet. v.372, n.9654, p.1988-96, 2008.

BRÊTAS, A.C.P.; GAMBA, M.A. Enfermagem e saúde do adulto. Barueri: Manole, 2006.

BUTTLER D. Un targets top killers. Nature. v.477, p. 260-1, 2011.

COELHO, C.F.; BURINI, R.C. Atividade física para prevenção e tratamento das doenças crônicas não transmissíveis e da incapacidade funcional. Revista de Nutrição, v.22, n.6, p.937946, 2009.

COSTA, A.P.; AFONSO, C.L.; DEMUNER, J.M.M.; MORAES, J.M.; PIRES, W.C. A importância da Liga acadêmica de queimaduras. Revista Brasileira de Queimaduras, v.8, n.3, p.101-105, 2009.

FEDERAÇÃO INTERNACIONAL DE DIABETES. 2009. Disponível em:<http://www.idf.org/webdata/docs/PR-IDFGlobalEducation201009_PT.pdf>. Acesso em:20/12/2016.

FERRARA, F.A. et al. Medicina de lacomunidad. Buenos Aires: Intermédia, 1972. 414 p.

GADAMER, H. G., 1994. Dove si Nascondela Salute. Milano: Raffaelo Cortina Editore.

GAMBA, M.A.; TADINI, A.C.O. Processo Saúde-Doença. Mimeografado, 2010.

GUARIGUATA, L. et al. The International Diabetes Federation Diabetes atlas methodology for estimating global and national prevalence of diabetes in adults. Diabetes, v.94, n.3, p.322-32, 2011. 
A liga acadêmica de clínica e cirurgia cardíaca: relato de experiência

HAMAMOTO FILHO, P.T.; VENDITTI, V.C.; OLIVEIRA, C.C.; VICENTINI, H.C.; SCHELLINI, S.A. Ligas Acadêmicas de Medicina: extensão das ciências médicas à sociedade. Revista Ciência em Extensão, v.7, n.1, p.126-133, 2011.

HAMAMOTO FILHO, P.T. Ligas Acadêmicas: motivações e críticas a propósito de um repensar necessário. Revista Brasileira de Educação Médica, v.35, n.4, p.535-543, 2011.

MARIATH, A.B. et al. Obesidade e fatores de risco para o desenvolvimento de doenças crônicas não transmissíveis entre usuários de unidade de alimentação e nutrição. Cadernos de Saúde Pública, v.23, n.4, p.897-905, 2007.

MONTEIRO, L.L.F.; CUNHA, M.S.; OLIVEIRA, W.L.; BANDEIRA, N.G.; MENEZES, J.V. Ligas Acadêmicas: O Que Há de Positivo? Experiência de Implantação da Liga Baiana de Cirurgia Plástica. Revista Brasileira de Cirurgia Plástica, v.23, n.3, p.158-161, 2008.

NEVES, F.B.C.S. et al. Inquérito nacional sobre as ligas acadêmicas de Medicina Intensiva. Revista brasileira de terapia intensiva, v.20, n.1, p.43-48, 2008.

OMS. ORGANIZAÇÃO MUNDIAL DA SAÚDE. Constituição da Organização Mundial da Saúde (OMS/WHO) - 1946.

PÊGO-FERNANDES, P.M.; MARIANI, A.W. O ensino médico além da graduação: ligas acadêmicas. Diagnóstico e Tratamento, v.16, n.2, p.50-51, 2011.

PEREIRA, L.O.; FRANCISCHI, R.P.; LANCHA JR, A.H. Obesidade: hábitos nutricionais, sedentarismo e resistência à insulina. Arquivos Brasileiros de Endocrinologia e Metabologia, São Paulo, v. 47, n. 2, p. 111-127, 2003.

PEREIRA, R.A.; ALVES-SOUZA R.A.; VALE, J.S. O processo de transição epidemiológica: uma revisão de literatura. Revista Científica da Faculdade de Educação e Meio Ambiente, v.6, n.1, p. 99-108, 2015.

SILVEIRA NETTO E. Atividade física para diabéticos. 1ª $^{\mathrm{a}}$ ed. Rio de janeiro: Sprint, 2000.

SOCIEDADE BRASILEIRA DE DIABETES. Diretrizes da Sociedade Brasileira de Diabetes. SP: AC Farmacêutica, 2015. Disponível em: http://www.diabetes.org.br/images/2015/area-restrita/diretrizes-sbd-2015.pdfAcesso em: 20/12/2016.

WHO. World Health Organization. Obesity: Data and Statistics, 2010. Disponívelem:http://www.euro.who.int/en/healthtopics/noncommunicablediseases/obesity/da ta-andstatistics. Acesso em: 20/12/2016

WHO. World Health Organization. The World Health Report 2005: health systems: improving performance. Geneva: WHO; 2005.

Recebido em: 16/09/2017

Aceito em: 27/07/2018 